\title{
Architecture Independent Analysis of Parallel Programs
}

\author{
Ananth Grama ${ }^{1}$, Vipin Kumar ${ }^{2}$, Sanjay Ranka ${ }^{3}$, and Vineet Singh ${ }^{4}$ \\ 1 Dept. of Computer Sciences, Purdue University, W. Lafayette, IN 47907 \\ ayg@cs . purdue .edu \\ 2 Dept. of Computer Sciences, University of Minnesota, Minneapolis, MN 55455 \\ kumar@cs . umn . edu \\ 3 Dept. of Computer Sciences, University of Florida, Gainesville, FL 32611 \\ ranka@cis.ufl.edu \\ 410535 Cordova Road, Cupertino, CA 95014
}

\begin{abstract}
The presence of a universal machine model for serial algorithm design, namely the von Neumann model, has been one of the key ingredients of the success of uniprocessors. The presence of such a model makes it possible for an algorithm to be ported across a wide range of uniprocessors efficiently. Universal algorithm design models proposed for parallel computers however tend to be limited in the range of parallel platforms they can efficiently cover. Consequently, portability of parallel programs is attained at the expense of loss of efficiency. In this paper, we explore desirable and attainable properties of universal models of architecture independent parallel program design. We study various models that have been proposed, classify them based on important machine parameters and study their limitations.
\end{abstract}

\section{Introduction}

Conventional sequential computing is based on the von Neumann model. This role of this model is illustrated in Figure 1. Given problem P, an optimal algorithm $\mathrm{O}$ is designed for solving it for model I. A description of this algorithm, along with a description of the architecture of the target machine $\mathrm{A}$ is fed into the translator $\mathrm{T}$. The translator generates machine code for the target machine. The set A spans the set of most conventional sequential computers and for this set, the von Neumann model fills in the role of model I.

In the above framework, the architecture independent algorithm design model I plays a very important role. It ensures that the same algorithm can be executed efficiently on a large variety of serial computers. Specifically, programs designed for this model have the property that they run in asymptotically the same time on every serial computer. The runtime of the algorithm on the said model is an accurate reflection of its asymptotic runtime on all computers subsumed by that model. The constants involved may not be reflected in the model but the model does provide a reasonable estimate. We refer to this as the predictive property of the model. Another interpretation of the predictive property is that 


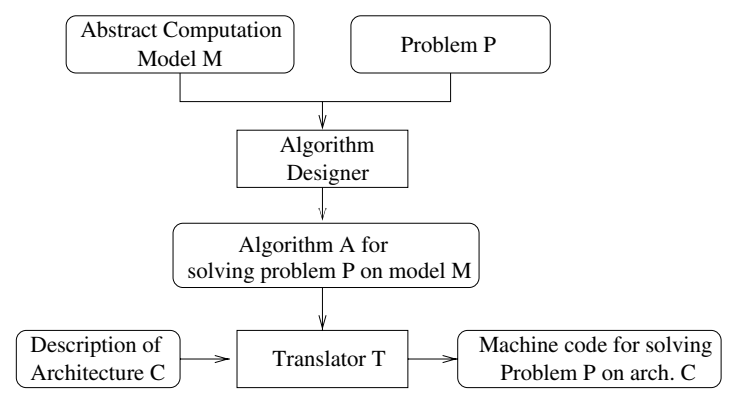

Fig. 1. The role of algorithm design model I in solving problem $\mathrm{P}$ on architecture A.

if two algorithms $\mathrm{O} 1$ and $\mathrm{O} 2$ are fed to the model, the model is able to predict which algorithm will have an asymptotically better performance on each computer subsumed by the model. We refer to this as the ordering property of the model. Finally, a translator exists that is capable of taking an algorithm specified for the model and converting it into a form that is executable by the target machine in asymptotically the same time as on model I. The translation process itself should not take an unreasonable amount of time. In the absence of such an architecture independent algorithm design model, we would have to design separate algorithms for each architecture.

The desirable properties of an architecture independent model do not change significantly in the context of parallel computers. However, due to added complexity, any such model can become difficult to program. It is therefore necessary to limit the complexity of the model. An architecture independent parallel programming model must have good coverage in architecture and algorithm space, be simple to work with, and have desirable performance prediction properties in the absolute, asymptotic, or relative (ordering) sense. It is unreasonable to expect accurate absolute (or even asymptotic) performance prediction from a model with good architecture and algorithm coverage. However, ordering properties are highly desirable for any proposed model.

\section{Taxonomy of Parallel Algorithm Design Models}

A number of models have been proposed that tradeoff accuracy, coverage, and simplicity to varying degrees. Whereas some models are relatively easy to design algorithms for, they may be difficult to realize in practice. In contrast, models that are closer to real machines tend to be less architecture independent and difficult to program. It is critical to identify various factors that differentiate the models and how they render models more or less realizable.

Global Memory Versus Seclusive Memories. The strongest models of algorithm design allow each word in a part of the memory to be addressed independent of all others (i.e., assume a global $p$-ported memory). Access to a certain word 


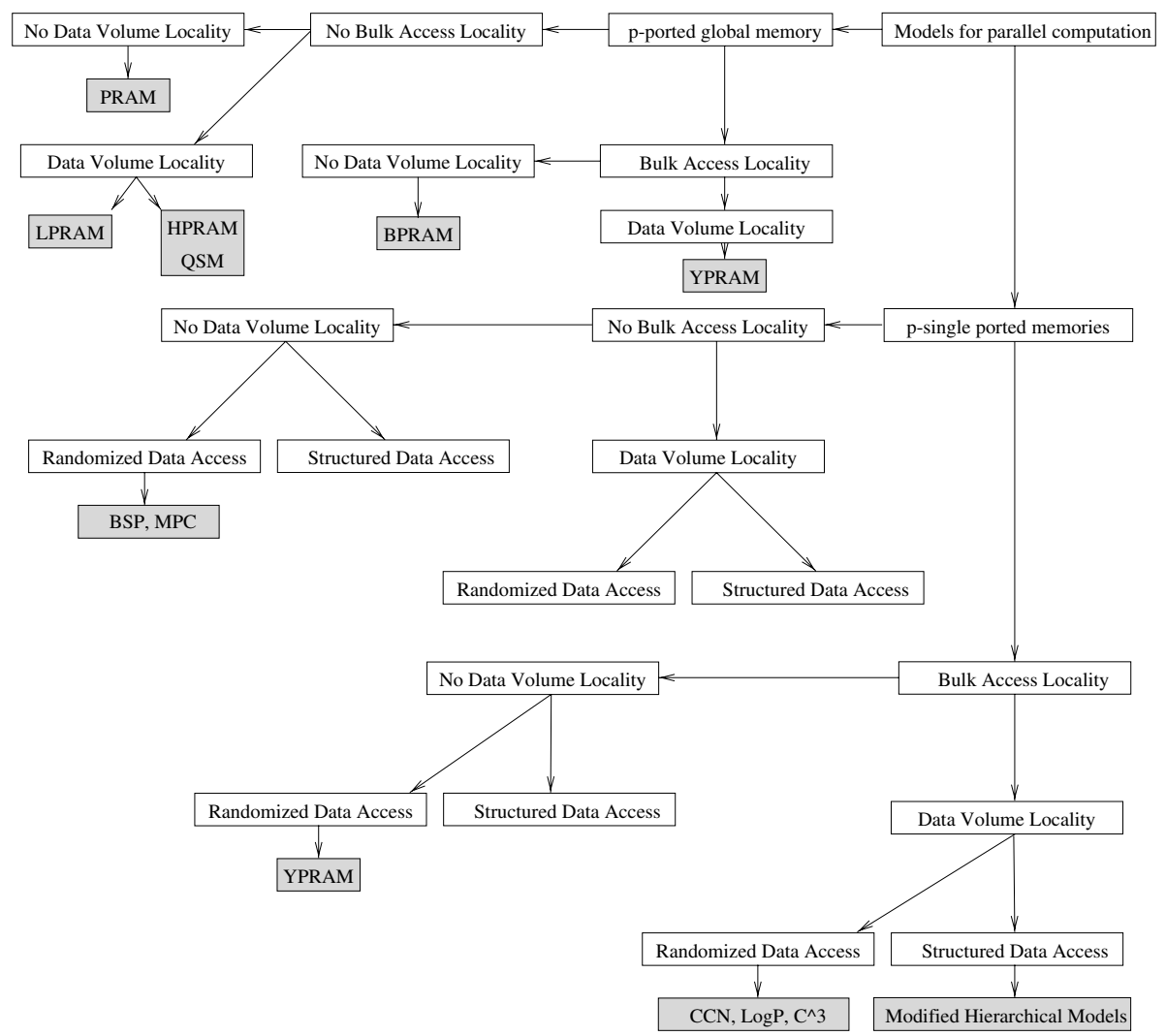

Fig. 2. Taxonomy of models for parallel programming.

in this global memory by a processor does not block access to any other word by another processor. Unfortunately, such a strong model comes with significant costs. If the total number of words in global memory is $m$ and the number of processors is $p$, a completely non-blocking switch has a complexity $\Theta(m p)$. Furthermore, the presence of processor caches imposes stronger requirements of the global memory. Most PRAM models (PRAM, LPRAM [3], BPRAM 44) assume a global memory.

The switch complexity can be reduced by organizing memory into banks ( $p$ banks of single-ported memories). When a processor is accessing a bank, access to all other words in the bank by other processors is blocked. If the switched memory is organized into $b$ banks, the switch complexity of a nonblocking (at the bank level) switch is $\Theta(p b)$. This complexity is realizable at the current state of the art. Such a model is referred to as a Seclusive Memory model. When combined with appropriate communication cost models, seclusive memory models approximate machine architectures much better than global 
memory models. Algorithm design models based on the DSM class of models include MPC, XPRAM[17], CCN, and $\log$ [5].

Bulk Access Locality. Bulk access locality requires accessing data in bulk due to non-negligible startup latencies. A dominant part of the startup latency is spent in packaging data, computing routing information, establishing protocols and programming routing hardware. This time varies significantly depending on the protocols used. Nevertheless, it is significantly higher than the processor cycle time in all cases. Although startup latency may not seem to be a major architecture parameter, it is in fact responsible, in significant part, for enforcing a degree of granularity in communication (and hence computation). Algorithm design models can be classified into those that reward bulk data access and those that do not. Models in the latter category are benign to algorithms that do not incorporate granularity. For such algorithms, the startup latency may have to be paid for each remote access on a real machine. Consequently, algorithms designed for such models perform poorly on real machines.

Data Volume Locality. The rate at which data can be fetched from local memory is generally much higher than that from a remote processor's memory. Efficient parallel algorithms must therefore minimize the volume of remote data accessed. This is referred to as data volume locality. Such locality is particularly important for platforms based on local area networks where the remote access rate (10s of $\mathrm{MB} / \mathrm{s})$ is much lower than local access rate $(100 \mathrm{~s}$ of $\mathrm{MB} / \mathrm{s})$. On many platforms, the degree of connectivity of the interconnection network is limited. For such platforms, the effective per-word transfer time for many permutations must be scaled up to accommodate the limited cross-section bandwidth. Machines with remote access time comparable to local memory access time, may have higher effective remote access times when compensated for cross-section bandwidth.

Even if it were possible to construct machines with inter-processor communication bandwidth matching local memory bandwidth, the presence of caches complicates the situation. Assuming a high cache hit ratio on serial computations, the effective local access time is the time to access the cache (and not the main memory). The inter-processor communication bandwidth must therefore match the local cache bandwidth. This is impossible to achieve without making bulk accesses. Therefore, even models that assume communication bandwidth equal to local memory bandwidth must incorporate bulk-access to mask cache effects and startup latency.

Structured Communication Operations. Typical applications can be viewed as a set of interacting subtasks executing on different processors. Interaction is by means of exchange of messages. It is often possible to formulate these tasks in such a way that the computation proceeds in interleaved steps of computation and communication. All processors (or subsets thereof) participate in the communication step. Since the communication involves more than a single pair of processors (aggregates or groups), such communication operations are also referred to as aggregate or collective communication operations. 
Most current parallel computers are capable of exploiting the structure of aggregate operations. However, many models ignore this structure and assume that all redistributions take equal time. A very common assumption made is that if the maximum amount of data entering or leaving a processor in a particular redistribution is $m$ then the communication time is proportional to $m$ and is independent of the number of processors $p$. This can be a misleading assumption. Consider a many-to-many personalized communication with fixed data fan-in and fan-out. This communication operation is also referred to as the transportation primitive [15]. It is possible to perform this operation in $\Theta(m)$ time ( $m$ is the data fan-in or fan-out) only in the presence of slack and $O(p)$ cross-section bandwidth [15. The accuracy of models making the above assumption can be poor and the class of real machines covered is relatively small.

There is a class of communication operations for which the optimal algorithms fit into a hierarchical framework. A hierarchical framework consists of hierarchies of redistributions. Redistributions involve only processors within a group. It is assumed that redistributions at various levels have no structure. Hierarchical models of parallel computers (YPRAM, PRAM) are capable of exploiting communication operations that fit into this hierarchical framework.

\section{Existing Models for Parallel Programming}

A variety of parallel algorithm design models have been proposed. They differ in their coverage, complexity, and accuracy.

LPRAM and BPRAM Models. The LPRAM [3] model of computing is a global memory model. It assumes that the startup latency is negligible. Both of these parameters are unrealizable in real machines of reasonable sizes. Furthermore, it does not reward structured data accesses. Algorithms designed for this model may potentially pay significant startup overheads and make poor use of available communication bandwidth. The bandwidth for remote access is assumed to be less than that for local memory access. Therefore, algorithms designed for LPRAMs reward only data volume locality.

The BPRAM [4] model assumes non-negligible startup latencies and a communication bandwidth identical to local memory bandwidth. It therefore rewards bulk access locality but fails to capture data volume locality. Machines conforming to this model are difficult to construct because of the presence of a global memory. Furthermore, if the processing nodes have a hierarchical memory structure, the effective bandwidth for local access is the bandwidth to cache (assuming a high hit ratio). The communication bandwidth must therefore match the bandwidth to cache in this model. This is difficult to realize unless communication is highly clustered.

Hierarchical RAM models (HPRAM, YPRAM, HMM). The HPRAM [1110] and YPRAM [6] models of computation allow recursive decomposition 
of the machine until the algorithm uses only one processor and executes sequentially. For more than one processor, the communication cost charged is a function of the number of processors available. Assigning different cost to data redistributions in which communication is limited to smaller subsets of processors allows the design of algorithms that are more effective in utilizing locality especially for divide and conquer type applications. Despite the availability of variable cost redistributions, the formulations described in [116] suffer from limitations similar to the BSP model (described subsequently) in terms of slack and worst case bandwidth requirements for every step. Analyzing time requirements using these models is difficult for non-recursively decomposable algorithms.

Bulk Synchronous Parallel and Related Models. The XPRAM 17 model allows PRAM algorithms to run within a constant factor of optimal as long as there is sufficient slack. The XPRAM model forms the basis for Bulk Synchronous Parallelism (BSP). It assumes a seclusive memory and for efficient operation, assumes startup latency to be negligible and per-word access time to be similar to cycle time. Although such a model is not as difficult to construct as global memory models, the restrictions on startup latency and per-word transfer time are difficult to realize in real machines. The main feature of BSP is that it allows simulation of $p \log p$ PRAM processors on $p$ seclusive memory processors in asymptotically the same time as on $p$ PRAM processors.

Although BSP [16] is an attractive theoretical model, it has several drawbacks. The cost of making a bulk access of $h$ words by a processor in a superstep is assumed to be $s+g^{\prime} h$. Here, $s$ is the startup cost and $g^{\prime}$ is the effective perword transfer time. For efficient simulation, this cost must be comparable to the cost of $h$ local memory accesses. This is difficult to achieve in realistic BSP simulations on actual platforms. The presence of hierarchical memory at processing nodes also requires bulk access locality. Even if the startup latency $t_{s}$ of the network was somehow reduced to a negligible amount, the time for realizing a $h$ relation must be similar to the cache access time for $h$ words on a uniprocessor (and not local memory access time). This can be achieved only through bulk access of data from processors.

The CGM model, proposed by Dehne [7, is an extension of the BSP model. It addresses the undesirable possibility in BSP of a large number of small messages. A CGM algorithm consists of alternating phases of computation and global communication. A single computation-communication pair corresponds to a BSP superstep and the communication phase corresponds to a single h-relation in BSP. However, in the case of CGM, the size of the h-relation is given by $h=n / p$, where $n$ corresponds to the problem size and $p$ to the number of processors. With this restriction on permutation size, the communication cost of a parallel algorithm is simply captured in the number of communication phases. This communication cost model, rewards a small number of larger messages, thus capturing spatial locality better than the BSP model.

Another extension of the BSP model proposed by Baumker et al. [2] also attempts to increase the granularity of messages by adding a parameter $B$, which 
is the minimum message size for the message to be bandwidth bound as opposed to latency bound. Messages of size less than $B$ are assigned the same cost as messages of size $B$, thus rewarding higher message granularity. The time taken by a BSP* $^{*}$ algorithm is determined by the sum of computation and communication supersteps. A computation superstep with $n$ local computations at each processor is assigned a cost $\max \{L, n\}$. A communication step with a h-relation of size $s$ is assigned a cost $\max \left\{g \times h \times\left\lceil\frac{s}{B}\right\rceil, L\right\}$. Thus algorithms aggregate messages into larger h-relations to utilize all available bandwidth.

CCN, LogP and Related Models. The Completely Connected Network (CCN) model of computing is a seclusive memory model. Although CCN has never been formally proposed as a model for architecture independent programming, its variants have been used extensively [12. It assumes the presence of $p$ processing elements connected over a completely connected network. The cost of sending a message of size $m$ from one processor to any other is given by $t_{s}+t_{w} m$. Here, $t_{s}$ is the startup latency and $t_{w}$ is the bandwidth term. It therefore rewards bulk access and data volume locality.

In many architectures, small, fixed size messages can be sent with a small overhead using active messages. This forms the basis for the $\operatorname{LogP}$ model of computation. In the LogP model processors communicate using small fixed size messages of size $m$. The model rewards bulk access at the level of small messages (using parameter $L$ ) and data volume locality (using parameter $g$ ). Larger messages are sent in LogP by breaking them into smaller messages and pipelining them. For each of the smaller messages, the processor incurs an overhead of $o$ at the sending end. The message can then be injected into the network. Subsequent messages can be injected at gaps of $g$. Here, $g$ is determined by the network bandwidth. If a sequence of short messages needs to be sent, the gap of the first message can be overlapped with the preparation overhead $o$ of the second message. Depending on values of $g$ and $o$, two situations arise: $g>o$ : in this case, the sending processor can prepare the messages faster than the network bandwidth. In this case, the network bandwidth will not be wasted. In the second case $o>g$ : in this case, the processor cannot inject messages into the network fast enough to utilize available bandwidth. Therefore, it is desirable that the size of the small fixed message $m$ be such that $g>o$. This implies that the model rewards bulk access to the level of $m$ words. Indeed, if $g>o$ and there is no overlap of computation and communication, this bulk is sufficient to mask startup latencies.

The postal model 1, which predates the $\log \mathrm{P}$ model, is a simplification of the LogP model. The postal model assumes a message passing system of $p$ processors with full connectivity (processors can send point-to-point messages to other processors), and simultaneous I/O (a processor can send one message and receive a message from another processor simultaneously). As is the case with $\log \mathrm{P}$, messages have a predefined size. Larger messages are composed as aggregates of these smaller messages. In a postal model with latency $\lambda$, a (small) message sent at time $t$ renders the sender busy for the time interval $[t, t+1]$ and 
the receiver busy for the interval $[t+\lambda-1, t+\lambda]$. Notice that this corresponds to the overhead $o$ of the $\log \mathrm{P}$ model being 1 and the latency $\lambda$ being identical to $L$.

The LogP model relies on short messages. However, several machines handle long messages much better than a sequence of short messages. In such cases, the $\log \mathrm{P}$ communication model is not very accurate. The LogGP model aims to remedy this by adding a parameter $G$, which captures the bandwidth for large messages. $G$ corresponds to the gap per byte (or data item) for large messages. In the $\log \mathrm{P}$ model, sending a $k$ item message takes time $o+(k-$ $1) * \max \{g, o\}+L+o$ cycles. In contrast, sending the message in the LogGP model (assuming the message is large) is given by $o+(k-1) G+L+o$ cycles. As before, the sending and receiving processors are busy only during the $o$ cycles of overhead and the rest of the time can be overlapped with useful computation. By reducing the overhead associated with large messages, LogGP rewards increased communication granularity.

These models suffer from drawbacks that stem from their inability to exploit structure in underlying communication patterns. They assume that all redistributions can be accomplished in time proportional to message size $(\Theta(m)$ for a $m$ word permutation). However, there are no known algorithms that can perform the permutation in this time even on $\Theta(p)$ cross-section bandwidth networks without slack. (The transportation primitive of Shankar and Ranka [15] performs this permutation in $\Theta(m)$ but it requires a total slack of $\Theta(p)$.) Therefore, the cost model is a lower bound for data redistributions on all architectures. Now consider an adaptation of these models for a lower connectivity network such as a 2-D mesh. The effective bandwidth is scaled down by the cross-section bandwidth to account for the limited bandwidth of the mesh while performing generalized redistributions. However, aggregate communication operations such as grid-communication and broadcasts consist entirely of redistributions that do not congest the network and are therefore capable of extracting the same network performance from a mesh as from a dense network such as a hypercube. In these cases, the communication cost of both models is an upper bound. The communication time predicted by the models is therefore neither an upper bound nor a lower bound. To remedy these problems the LogP model itself suggests use of different values of gap $g$ for different communication patterns, however, it does not provide details on how to do so.

Contention Modeling Abstractions. The Queuing Shared Memory Model (QSM) of Ramachandran et al. 13 also follows a bulk synchronous format with phases of shared memory reads, shared memory writes, and local computation. The performance model of QSM explicitly accounts for remote memory accesses, contention, and congestion. The primary parameters in the QSM model include the number of processors $p$ and the remote data access rate in terms of local instruction cycles $g$. If in a phase, a parallel algorithm executes $m_{o p}$ local memory operations and $m_{r w}$ remote operations, with a contention of $\kappa$, QSM associates a cost $\max \left\{m_{o p}, g \cdot m_{r w}, \kappa\right\}$ with it. Minimizing this maximizes local data access 
and minimizes contention to shared data. In addition to these, QSM also has a set of secondary parameters - latency $l$, barrier time $L$, message sending overhead $o$, memory bank contention $h_{r}$, and network congestion $c$. The process of algorithm design can be largely simplified by considering only the primary parameters and optimizing the secondary factors using known techniques such as pipelining for latency hiding, randomization for bank conflicts, etc.

The $\mathrm{C}^{3}$ model, proposed by Hambrusch and Khokhar 9 abstracts a $p$ processor machine in terms of the communication packet size $l$, the setup cost for a message $s$, communication latency $h$, and bisection width $b$. A parallel algorithm is assumed to proceed in supersteps of computation and communication. The cost of the computation phase of a superstep is determined by the maximum number of data items accessed by any processor (expressed in terms of number of packets). The cost of the communication phase is determined by whether the communication is blocking or non-blocking. A blocking communication of $m$ packets is charged a time $2(s+h)+m+h$ at the sender and $s+2 h+m$ at the receiver. A non-blocking communication of $m$ packets is charged a time $s+m+h$ at the sender and $m$ at the receiver. Communication congestion is modeled in terms of network congestion and processor congestion. This is determined by the number of processor pairs communicating $c$, and the average number of packets $r$ being communicated by any pair. The network congestion $C_{l}$ is given by $C_{l}=(r \times c) / b$ and the processor congestion $C_{p}$ by $C_{p}=(r \times c \times h) / p$. The overall communication cost is the maximum of sender time, receiver time, processor congestion, and network congestion.

While both of these models are refinements of BSP and LogP models, they suffer from many of the same drawbacks as $\log \mathrm{P}$ and BSP models.

The $\mathrm{A}^{3}$ model 8 expresses communication cost as a combination of the architecture and underlying permutation. It classifies commonly used communication operations into those that are sensitive to the bisection bandwidth, and those that are not. It then uses the bisection width of the network to derive appropriate costs for aggregate communication operations. Algorithm analysis is reduced to the process of a table lookup for accurate or approximate cost associated with each operation. It is shown that this model has good coverage in algorithm in architecture space and has excellent predictive properties.

\section{Concluding Remarks}

In this paper, we presented a brief survey of prominent parallel algorithm design models. As is evident, in spite of extensive research, the search for a universal parallel algorithm design model has had only limited success. Advances in this area have tremendous significance, not just for parallel architectures, but also for serial processors with deep memory hierarchies.

\section{References}

1. A. Bar-Noy and S. Kipnis. Designing Broadcasting Algorithms in the Postal Model for Message-Passing Systems. Proceedings of 4-th ACM Symp. on Parallel Algorithms and Architectures, pp. 13-22, 1992. 
2. Armin Bumker, Wolfgang Dittrich, Friedhelm Meyer auf der Heide. Truly Efficient Parallel Algorithms: 1-optimal Multisearch for an Extension of the BSP Model. Theoretical Computer Science, 203(2): 175-203, 1998.

3. A. Agarwal, A. K. Chandra, and M. Snir. Communication complexity of PRAMs. Technical Report RC 14998 (No.64644), IBM T.J. Watson Research Center, Yorktown Heights, NY, 1989.

4. A. Agarwal, A. K. Chandra, and M. Snir. On communication latency in PRAM computations. Technical Report RC 14973 (No.66882), IBM T.J. Watson Research Center, Yorktown Heights, NY, 1989.

5. D. Culler, R. Karp, D. Patterson, A. Sahay, K.E. Schauser, E. Santos, R. Subramonian, and T. von Eicken. LogP: Towards a Realistic Model of Parallel Computation. Proceedings of 4-th ACM SIGPLAN Symp. on Principles and Practices of Parallel Programming, pp. 1-12, 1993.

6. Pilar de la Torre and Clyde P. Kruskal. Towards a single model of efficient computation in real parallel machines. In Future Generation Computer Systems, pages $395-408,8(1992)$.

7. F. Dehne, A. Fabri, and A. Rau-Chaplin. Scalable parallel computational geometry for coarse grained multicomputers. International Journal on Computational Geometry, Vol. 6, No. 3, 1996, pp. 379 - 400.

8. Ananth Grama, Vipin Kumar, Sanjay Ranka, and Vineet Singh. A ${ }^{3}$ : A simple and asymptotically accurate model for parallel computation. In Proceedings of Conference on Frontiers of Massively Parallel Computing, page 8 pp, Annapolis, MD, 1996.

9. S. Hambrusch and A. Khokhar. $\mathrm{C}^{3}$ : A parallel model for coarse-grained machines. Journal of Parallel and Distributed Computing, 32(2):139-154, Feb 1996.

10. T. Heywood and S. Ranka. A practical hierarchical model of parallel computation. i. the model. Journal of Parallel and Distributed Computing, 16(3):212-32, Nov. 1992.

11. T. Heywood and S. Ranka. A practical hierarchical model of parallel computation. ii. binary tree and fft algorithms. Journal of Parallel and Distributed Computing, 16(3):233-49, Nov. 1992.

12. Vipin Kumar, Ananth Grama, Anshul Gupta, and George Karypis. Introduction to Parallel Computing: Algorithm Design and Analysis. Benjamin Cummings/ Addison Wesley, Redwod City, 1994.

13. Vijaya Ramachandran. QSM: A General Purpose Shared-Memory Model for Parallel Computation. FSTTCS 1-5, 1997.

14. A. G. Ranade. How to emulate shared memory. In Proceedings of the 28th IEEE Annual Symposium on Foundations of Computer Science, pages 185 - 194, 1987.

15. R. Shankar and S. Ranka. Random data access on a coarse grained parallel machine ii. one-to-many and many-to-one mappings. Technical report, School of Computer and Information Science, Syracuse University, Syracuse, NY, 13244, 1995.

16. L. G. Valiant. A bridging model for parallel computation. Communications of the ACM, 33(8), 1990.

17. L. G. Valiant. General purpose parallel architectures. Handbook of Theoretical Computer Science, 1990.

18. J. S. Vitter and E. A. M. Shriver. Algorithms for Parallel Memory II: Hierarchical Multilevel Memories. newblock Algorithmica, 12(2-3), 1994, 148-169.

19. T. von Eicken, D. E. Culler, S. C. Goldstein, and K. E. Schauser. Active messages: a mechanism for integrated communication and computation. In Proceedings of the 19th International Symposium on Computer Architecture, 1992. 